\title{
Actividad microbiana en suelos de sabanas de los Llanos Orientales de Venezuela convertidas en pasturas
}

\author{
Y. Gómez ${ }^{1} \&$ J. Paolini² \\ 1 Universidad de Oriente, Núcleo de Anzoátegui, Laboratorio de Investigaciones Biológicas, Apdo. 1231, Puerto La \\ Cruz, Estado Anzoátegui, Venezuela. Tlf:+58-2812-2819239; irmagomez52@hotmail.com \\ 2 Instituto Venezolano de Investigaciones Científicas (IVIC), Centro de Ecología, Apdo. 21827, Caracas 1020-A, \\ Venezuela. Tlf: +58- 0212-504-1280; jpaolini@ivic.ve
}

Recibido 20-VI-2001. Corregido 27-VII-2002. Aceptado 30-IV-2003.

\begin{abstract}
Microbial activity in Eastern Venezuelan savannas turned into grasslands. We studied the effects of season and management practices on chemical properties and biochemical parameters in a typical Ultisol soil of native and introduced pastures of the Eastern savannas of Venezuela. Sseason, soil management, and their interaction, significantly affected chemical properties and biochemical parameters. The total carbon and the water soluble carbon experienced a significant decrease during the wet pereiod. The basal respiration, the arginine ammonification (AA) and the fluoriscein diacetate hydrolysis showed showed a relatively low microbial activity. The transformation of native savannas to pastures systems of B. brizantha and S. capitata increased the organic matter content in soil. The AA and the basal respiration were sensible indicators to estimate changes in soil quality and also reflected the interaction of temporal changes and management practices. Rev. Biol. Trop. 54(2): 273-285. Epub 2006 Jun 01.
\end{abstract}

Key words: arginine ammonification, basal respiration, fluoriscein diacetate, management practices, savannas, temporal variability, Venezuela.

Los microorganismos del suelo llevan a cabo una variedad de funciones, las cuales son necesarias para la salud del mismo (Kennedy y Papendick 1995, Sparling 1997). Estos degradan las sustancias orgánicas a través de diversas actividades metabólicas que determinan el almacenamiento y reciclaje de los nutrientes en el suelo (Srivastava y Singh 1988). Estas actividades resultan severamente afectada por varios factores, entre ellos la variabilidad climática. Durante la época de sequía, los microorganismos están sujetos al estrés hídrico, lo cual afecta el metabolismo celular (Coyne 2000); efecto que repercute directamente sobre el reciclaje de la materia orgánica en suelo (Sparling y West 1989). Otro factor que afecta a las poblaciones microbianas y sus actividades, es el manejo agrícola, dentro del cual la agricultura intensiva ha conducido a la degradación acelerada del suelo (Kieff 1994, Lovell et al. 1995, Delgado 2001), provocando la pérdida del contenido de la materia orgánica en éste y por ende su calidad (Doran y Parkin 1994, Fauci y Dick 1994).

Se ha tratado de establecer aquellas propiedades del suelo que resultan más sensibles a los cambios provocados por las prácticas de manejo (Elliot 1994) y su selección ha sido enfocada sobre aquellos parámetros que se espera cambien más rápidamente (Dick 1994, Leiros et al. 2000), discriminen entre los efectos de varias prácticas de manejo (Fauci y Dick 1994), que muestren sensibilidad al estrés y restauración del suelo (Dick y Tabatabai 1992, Dick 1997), y que reflejen en forma segura los cambios espaciales y temporales (Kennedy y 
Papendick 1995). Dentro de éstos se encuentran los parámetros bioquímicos.

A pesar de la importancia de los parámetros bioquímicos para hacer comparaciones entre la calidad de los ecosistemas nativos y aquellos modificados por el manejo agrícola (Sparling 1997), las investigaciones en este campo en las sabanas tropicales son escasas.

En este sentido, en esta investigación se seleccionaron como parámetros bioquímicos: la respiración basal (RB) (Anderson 1982, Brookes 1985), la amonificación de la arginina (AA) (Alef y Kleiner 1987, Gil Sotres, 1997, Torben et al. 2001) y la hidrólisis del diacetato de fluorisceína (DAF) (Schnürer y Rosswall, 1982, Dick 1994), ya que éstos reflejan la actividad microbiana heterotrófica en el suelo, con el objeto de determinar el efecto de la variación estacional y el manejo del agrícola sobre estos y algunas propiedades químicas de suelos de las sabanas de los Llanos Orientales, e identificar aquellos parámetros que pudieran servir como indicadores de cambios en calidad del suelo.

\section{MATERIALES Y MÉTODOS}

Área de estudio. El experimento se llevó a cabo en las áreas experimentales de Rancho Comanche (RC) y la estación experimental del Instituto Nacional de Investigaciones Agropecuarias (INIA) en el Estado Anzoátegui, Venezuela, los cuales están ubicados en el Municipio Simón Rodríguez ( $8^{\circ} 47^{\prime}$ N, 64 $13^{\prime} \mathrm{W}$ ) y en el Municipio San José de Guanipa ( $8^{\circ} 52^{\prime}$ $\mathrm{N}, 64^{\circ} 13^{\prime} \mathrm{W}$ ), respectivamente, a una altitud entre 250 y 300 m.s.n.m. La temperatura máxima anual es de $32^{\circ} \mathrm{C}$ y la mínima de $22^{\circ} \mathrm{C}$, con pequeñas variaciones mensuales. Estas presentan dos períodos estacionales bien definidos, uno de sequía comprendido entre Diciembre y Mayo, y otro lluvioso entre Junio y Noviembre, con precipitaciones que varían entre los 1000-1200 mm por año (MARNR 1997, Romero 1979). El promedio de evaporación es de $2700 \mathrm{~mm}$ (Albornoz et al. 1995). El elemento geomorfológico de las áreas seleccionadas lo constituyen las Mesas y su basamento geomorfológico es la Formación Mesa de edad cuaternaria (Marrero 1964). Sus suelos se caracterizan por tener textura arenosa, clasificados como Ultisoles (Kandiustul) (Navas 2000), presentan pH ácido (4.8-5.7), bajo contenido de carbono (2.06-4.39 $\mathrm{g} \mathrm{kg}^{-1} \mathrm{~s}$ ) y una CIC efectiva de 3 meq $100 \mathrm{~g}^{-1} \mathrm{~s}$.

Historia del cultivo. La parcela experimental de RC fue sometida a labranza (arado y rastra) y cultivado con maní (Arachis hypogaea) durante 15 años. En 1981 fue sometido nuevamente a la labranza (arado y rastra) antes del establecimiento del pasto suazi (Digitaria swazilandensis) por 15 años. Luego en 1995 se introdujo la cobertura de B. brizantha, para lo cual el sitio fue nuevamente sometido al mismo sistema de labranza.

$\mathrm{Al}$ igual que en RC, en el INIA, se empleó arado y rastra en 1998, antes de la introducción de la cobertura Centrosema rotundifolium establecida hasta el 2000, fecha a partir del cual el área fue nuevamente sometida a este tipo de labranza para introducir la leguminosa forrajera $S$. capitata. Ambas parcelas han recibido fertilización inorgánica con 12:24:12 (NPK), reabonos con urea y encalado del suelo durante el inicio del período lluvioso.

Muestreo. El muestreo del suelo se realizó durante la estación seca (Marzo del 2001) y el período lluvioso (Julio de 2001). En cada área experimental, el muestreo se llevó a cabo en 2 parcelas de $100 \times 20 \mathrm{~m}^{2}$ cercanas entre sí; una ubicada en la sabana nativa y otra en la intervenida. Las muestras fueron recolectadas con un barreno entre $0-10 \mathrm{~cm}$ de profundidad a lo largo de tres transectos, cada uno con 5 puntos de muestreo, distribuidos a intervalos regulares de $20 \mathrm{~m}$. Las muestras obtenidas en cada transecto fueron mezcladas homogéneamente para formar una muestra compuesta, obteniéndose así tres compuestas por parcelas. Las muestras se tomaron a humedad de campo y se colocaron en bolsas plásticas para prevenir su secado. Una vez en el laboratorio, estas fueron cernidas $(<2 \mathrm{~mm})$ y almacenadas a $4^{\circ} \mathrm{C}$ hasta la determinación de las propiedades químicas y los parámetros bioquímicos correspondientes. Los análisis se realizaron por triplicado. 
Análisis químico del suelo. El contenido de humedad fue determinado mediante un analizador de humedad Ohaus a $105^{\circ} \mathrm{C}$ y el $\mathrm{pH}$ del suelo fue medido electrométricamente en una suspensión de agua (1:2.5) (p:v). El carbono orgánico total (COT) del suelo se determinó espectrofotométricamente, mediante el método de oxidación de Walkley y Black (1934). La determinación del Carbono hidrosoluble (CHS), se realizó en un analizador de carbono Shimadzu, modelo TOC-500.

\section{Métodos analíticos}

Respiración basal. La respiración basal se determinó según el método descrito por Alef y Nannipieri (1995). Las muestras de suelo a humedad de campo y cernidas $(<2 \mathrm{~mm})$ fueron preacondicionadas por 5 días a temperatura del laboratorio $\left(24^{\circ} \mathrm{C}\right)$ para lograr su estabilización.

$50 \mathrm{~g}$ de suelo fueron colocados en frascos de vidrio del $500 \mathrm{ml}$ de capacidad, se sellaron herméticamente y se incubaron por $24 \mathrm{hr}$. El $\mathrm{CO}_{2}$ liberado fue atrapado en una solución de $\mathrm{NaOH}(0.01 \mathrm{~N}) . \mathrm{El} \mathrm{CO}$ absorbido fue titulado con HCL $(0.01 \mathrm{~N})$ después de la adición de $2 \mathrm{ml} \mathrm{de} \mathrm{BaCl}_{2}$ y dos gotas de fenoftaleína (Šantrùèková 1991). Frascos vacíos con trampas de álcali se emplearon para chequear el contenido del $\mathrm{CO}_{2}$ del aire. Los resultados fueron expresados en mg C-CO $\mathrm{kg}^{-1} 24 \mathrm{hr}^{-1}$.

Amonificación de la arginina. La amonificación de la arginina (AA) fue determinada por triplicado según el método descrito por Alef y Kleiner (1986). A $2 \mathrm{~g}$ de suelo a humedad de campo se le añadieron gota a gota, $0.5 \mathrm{ml}$ de una solución de arginina ( $0.2 \%$ en agua). Las muestras fueron incubadas por $4 \mathrm{hr}$ a $37^{\circ} \mathrm{C}$ y extraídas con $8 \mathrm{ml}$ de $\mathrm{KCl}(2 \mathrm{M})$. Luego fueron agitadas por $30 \mathrm{~min}$ y centrifugadas a $3000 \mathrm{rpm}$ por $10 \mathrm{~min}$.

El amonio liberado en cada extracto fue determinado colorimétricamente a $600 \mathrm{~nm}$ por el método del nitroprusiato de sodio de Dorich y Nelson (1983). Los resultados fueron expresados en $\mu \mathrm{g} \mathrm{N}-\mathrm{NH}_{4}^{+} \mathrm{g}^{-1} \mathrm{hr}^{-1}$.
Hidrólisis del diacetato de fluorisceína. La hidrólisis del diacetato de fluorisceína (DAF) se determinó por triplicado según el método descrito por Gillian y Duncan (2001). El cual consiste en añadir a $2 \mathrm{~g}$ de suelo fresco, $15 \mathrm{ml}$ del buffer fosfato de potasio $(60 \mathrm{mM})$ pH 7.6 y $0.2 \mathrm{ml}$ de una solución de DAF (1000 $\mu \mathrm{g} \mathrm{ml}^{-1}$ ) como sustrato. Los blancos se prepararon sin la adición del DAF. Los frascos fueron tapados, agitados e incubados a $30^{\circ} \mathrm{C}$ por $1 \mathrm{hr}$. Concluido este período, a cada una de las muestras se les añadió $15 \mathrm{ml}$ de una solución de cloroformo/metanol $(2: 1 \mathrm{v} / \mathrm{v})$ para detener la reacción. Las muestras fueron agitadas y centrifugadas a $2000 \mathrm{rpm}$ por $3 \mathrm{~min}$. El sobrenadante de cada muestra fue filtrado (Whatman $\mathrm{N}^{\circ} 42$ ), y el filtrado fue medido a $490 \mathrm{~nm}$. Los resultados fueron expresados en $\mu \mathrm{g}$ de fluorisceína $\mathrm{g}^{-1} \mathrm{hr}^{-1}$.

Análisis estadísticos. Los datos fueron analizados mediante la prueba de t-student para determinar las diferencias entre las medias provocadas por efecto de la variabilidad temporal y el manejo del suelo, y el análisis de ANOVA de dos vías para determinar el efecto interactivo entre ambos factores. Los análisis estadísticos se realizaron a través del programa estadístico SPSS para Windows (Vinacua 1997).

\section{RESULTADOS}

Las propiedades químicas y los parámetros bioquímicos específicos de los suelos de las sabanas de los Llanos Orientales de Venezuela mostraron variaciones como consecuencia de la condición climática, el manejo del suelo y la interacción de ambos factores (Cuadros 1, 2 y 3).

Propiedades químicas. El Cuadro 1 expresa las variabilidad temporal de las propiedades químicas del suelo de las sabanas nativas e intervenidas de RC y el INIA.

$\mathrm{El} \mathrm{pH}$ del suelo experimentó un incremento significativo durante el período lluvioso, lo cual se explica por el efecto de la dilución (Buckman y Brady, 1966 Fassbender y Bornemiza 1987). En contraste, a excepción 


\section{CUADRO 1}

Efecto de la variabilidad temporal sobre algunas propiedades químicas de suelos de sabanas de los Llanos Orientales de Venezuela, en condiciones de vegetación natural y pasturas

\section{TABLE 1}

Effect of temporal variability on some chemical properties of savanna soils, Venezuela Eastern Llanos, under native vegetation and pasture systems

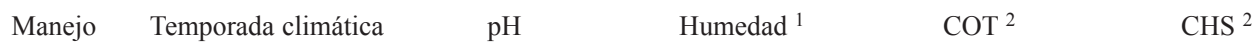

$\mathrm{RC}$

$\begin{array}{llllll}\text { Trachypogon } & \text { Sequía } & 4.96 \pm 0.04^{\mathrm{a}} & 1.0 \pm 0.0^{\mathrm{a}} & 2.71 \pm 0.045^{\mathrm{a}} & 0.10 \pm 0.07^{\mathrm{a}} \\ \text { Trachypogon } & \text { Lluvia } & 5.62 \pm 0.07^{\mathrm{b}} & 3.88 \pm 0.03^{\mathrm{b}} & 2.89 \pm 0.044^{\mathrm{b}} & 0.06 \pm 0.002^{\mathrm{b}} \\ \begin{array}{l}\text { B. } \text { brizantha }^{\mathrm{a}} \\ \text { B. brizantha }\end{array} & \text { Sequía } & 5.41 \pm 0.1^{\mathrm{a}} & 1.41 \pm 016^{\mathrm{a}} & 4.39 \pm 0.137^{\mathrm{a}} & 0.15 \pm 0.05^{\mathrm{a}} \\ \text { INIA } & \text { Lluvia } & 5.76 \pm 0.05^{\mathrm{b}} & 2.18 \pm 0.21^{\mathrm{b}} & 3.31 \pm 0.065^{\mathrm{b}} & 0.13 \pm 0.005^{\mathrm{b}} \\ \text { Trachypogon } & & & & \\ \text { Trachypogon } & \text { Sequía } & 4.81 \pm 0.04^{\mathrm{a}} & 1.36 \pm 0.2^{\mathrm{a}} & 3.94 \pm 0.087^{\mathrm{a}} & 0.15 \pm 0.002^{\mathrm{a}} \\ \text { S. capitata } & \text { Lluvia } & 5.31 \pm 0.05^{\mathrm{b}} & 1.70 \pm 0.2^{\mathrm{a}} & 2.75 \pm 0.115^{\mathrm{b}} & 0.11 \pm 0.005^{\mathrm{b}} \\ \text { S. capitata } & \text { Sequía } & 4.86 \pm 0.04^{\mathrm{a}} & 0.84 \pm 0.05^{\mathrm{a}} & 4.12 \pm 0.118^{\mathrm{a}} & 0.21 \pm 0.003^{\mathrm{a}} \\ & \text { Lluvia } & 5.21 \pm 0.05^{\mathrm{b}} & 1.95 \pm 0.02^{\mathrm{b}} & 2.06 \pm 0.109^{\mathrm{b}} & 0.12 \pm 0.003^{\mathrm{b}}\end{array}$

Los valores medios acompañados del error y seguidos de la misma letra no difieren significativamente entre si.

1. $\%$ • 2. $\mathrm{g} \mathrm{Kg}^{-1}$

\section{CUADRO 2}

Efecto de la variabilidad temporal sobre parámetros bioquímicos de suelos de sabanas de los Llanos Orientales de Venezuela en condiciones de vegetación natural y pastura

TABLE 2

Effect of temporal variability on biochemical parameters of savanna soils, Venezuela Eastern Llanos, under native vegetation and pasture systems
Manejo
Temporada climática
$\mathrm{RB}^{1}$
$\mathrm{AA}^{2}$
$\mathrm{DAF}^{3}$

$\mathrm{RC}$

\begin{tabular}{|c|c|c|c|c|}
\hline Trachypogon & Sequía & $7.54 \pm 0.7^{\mathrm{a}}$ & $1.07 \pm 0.04^{\mathrm{a}}$ & $38.65 \pm 3.0^{\mathrm{a}}$ \\
\hline Trachypogon & Lluvia & $15.86 \pm 0.7^{\mathrm{b}}$ & $3.66 \pm 0.12^{b}$ & $51.07 \pm 8.0^{\mathrm{b}}$ \\
\hline B. brizantha & Sequía & $7.54 \pm 0.7^{\mathrm{a}}$ & $1.61 \pm 0.05^{\mathrm{a}}$ & $40.76 \pm 1.5^{\mathrm{a}}$ \\
\hline B. brizantha & Lluvia & $16.83 \pm 0.7^{\mathrm{b}}$ & $2.10 \pm 0.05^{\mathrm{b}}$ & $44.70 \pm 2.4^{b}$ \\
\hline \multicolumn{5}{|l|}{ INIA } \\
\hline Trachypogon & Sequía & $8.03 \pm 0.7^{\mathrm{a}}$ & $0.99 \pm 0.04^{\mathrm{a}}$ & $41.05 \pm 2.1^{\mathrm{a}}$ \\
\hline Trachypogon & Lluvia & $13.41 \pm 0.5^{b}$ & $1.74 \pm 0.03 \mathrm{~b}$ & $45.64 \pm 3.0^{\mathrm{b}}$ \\
\hline S. capitata & Sequía & $11.94 \pm 0.8^{\mathrm{a}}$ & $1.38 \pm 0.04^{\mathrm{a}}$ & $47.41 \pm 3.4^{\mathrm{a}}$ \\
\hline S. capitata & Lluvia & $23.68 \pm 1.0^{b}$ & $1.88 \pm 0.02 \mathrm{~b}$ & $53.35 \pm 5.1^{b}$ \\
\hline
\end{tabular}

Los valores medios acompañados del error y seguidos de la misma letra no difieren significativamente entre si. 1. $\mathrm{mg} \mathrm{C}_{-} \mathrm{CO}_{2} \mathrm{Kg}^{-1} \mathrm{~s} 24 \mathrm{~h}^{-1} \cdot 2$. $\mu \mathrm{g} \mathrm{N}-\mathrm{NH}_{4}^{+} \mathrm{g}^{-1} \mathrm{~h}^{-1} \bullet 3$. $\mu$ g Fluoriscina $\mathrm{g}^{-1} \mathrm{~h}^{-1}$ 
CUADRO 3

Diferencias estadísticas (valores de F y niveles de significancia) entre las medias de las propiedades químicas y parámetros bioquímicos

TABLE 3

Statistical differences ( $F$ values and significance levels) between means of chemical properties and biochemical parameters

\begin{tabular}{|c|c|c|c|c|}
\hline Variables & Área & Estación & Manejo & Interacción \\
\hline \multirow[t]{2}{*}{$\mathrm{pH}$} & R.C. & $50.112 * * *$ & $16.998 * * *$ & $5.089 *$ \\
\hline & INIA & $89.494 * * *$ & $0.377^{\mathrm{NS}}$ & $2.551^{\mathrm{NS}}$ \\
\hline \multirow[t]{2}{*}{ Humedad ${ }^{1}$} & R.C. & $186.679 * * *$ & $23.453 * * *$ & $62.751 * * *$ \\
\hline & INIA & $28.167 * * *$ & $0.913^{\mathrm{NS}}$ & $7.761 * *$ \\
\hline \multirow[t]{2}{*}{$\mathrm{COT}^{2}$} & R.C. & $162.693 * * *$ & $30.733 * * *$ & $59.007 * *$ \\
\hline & INIA & $5.584 *$ & $223.259 * * *$ & $15.967 * * *$ \\
\hline \multirow[t]{2}{*}{$\mathrm{CHS}^{2}$} & R.C. & $62.676 * * *$ & $235.488 * * *$ & $6.328 *$ \\
\hline & INIA & 361.092 *** & $80.496 * * *$ & $36.483 * * *$ \\
\hline \multirow[t]{2}{*}{$\mathrm{RB}^{3}$} & R.C. & $132.923 * * *$ & $0.410^{\mathrm{NS}}$ & $0.410^{\mathrm{NS}}$ \\
\hline & INIA & $125.641 * * *$ & $86.256 * * *$ & $17.333 * * *$ \\
\hline \multirow[t]{2}{*}{$\mathrm{AA}^{4}$} & R.C. & 456.239 *** & $50.914 * * *$ & $214.396 * * *$ \\
\hline & INIA & $374.531 * * *$ & $65.416 * * *$ & $14.143 * *$ \\
\hline \multirow[t]{2}{*}{$\mathrm{DAF}^{5}$} & R.C. & $29.283 * * *$ & 1.977 NS & $7.859 * *$ \\
\hline & INIA & $19.759 * * *$ & $35.269 * * *$ & $0.322^{\mathrm{NS}}$ \\
\hline
\end{tabular}

de la sabana nativa de RC, el COT del suelo disminuyó durante la temporada lluviosa. Éste varió en la sabana nativa de RC de 2.71 a $2.89 \mathrm{~g}$ $\mathrm{kg}^{-1}$, y en la sabana intervenida bajo la pastura de $B$. brizantha la variación fue de $4.39-3.31 \mathrm{~g}$ $\mathrm{kg}^{-1}$; mientras que en el INIA, esta variación en la sabana nativa fue de 3.94 a $2.75 \mathrm{~g} \mathrm{~kg}^{-1} \mathrm{y}$ de $4.12-2.06 \mathrm{~g} \mathrm{~kg}^{-1}$ en la sabana bajo la cobertura de $S$. capitata.

El carbono hidrosoluble (CHS) del suelo también experimentó una disminución significativa durante la temporada lluviosa en ambas áreas de estudio (Cuadro1). Esta fracción del carbono en las sabanas nativas de $\mathrm{RC}$ y el INIA, varió de $0.10-0.06 \mathrm{~g} \mathrm{~kg}^{-1}$ y de 0.15 $-0.11 \mathrm{~g} \mathrm{~kg}^{-1}$, respectivamente, y en las sabanas bajo la cobertura de $B$. byzantha y $S$. apitata la variación fue de $0.15-0.13 \mathrm{~g} \mathrm{~kg}^{-1}$ y de $0.21-$ $0.12 \mathrm{~g} \mathrm{~kg}^{-1}$, respectivamente. Así mismo, el manejo agrícola provocó variaciones significativas $(\mathrm{p}<0.001)$ en el contenido de COT y CHS en el suelo de las sabanas de RC y el INIA (Figs. 1 A y B). En RC durante los períodos de lluvia y sequía, y bajo la cobertura de $B$. brizantha, el COT incrementó con respecto a la sabana nativa en un $38.26 \%$ y en un $12 \%$, respectivamente. Mientras que en el INIA, bajo la cobertura de $S$. capitata, el COT incrementó con respecto a la sabana nativa durante la temporada seca en un 4.36\%; no obstante, en el período lluvioso, éste disminuyó de $25.1 \%$ (Fig. 1 A).

En RC bajo la cobertura de B. brizantha, el CHS incrementó en un $33.3 \%$ durante la estación seca y en un $53.84 \%$ durante el período 

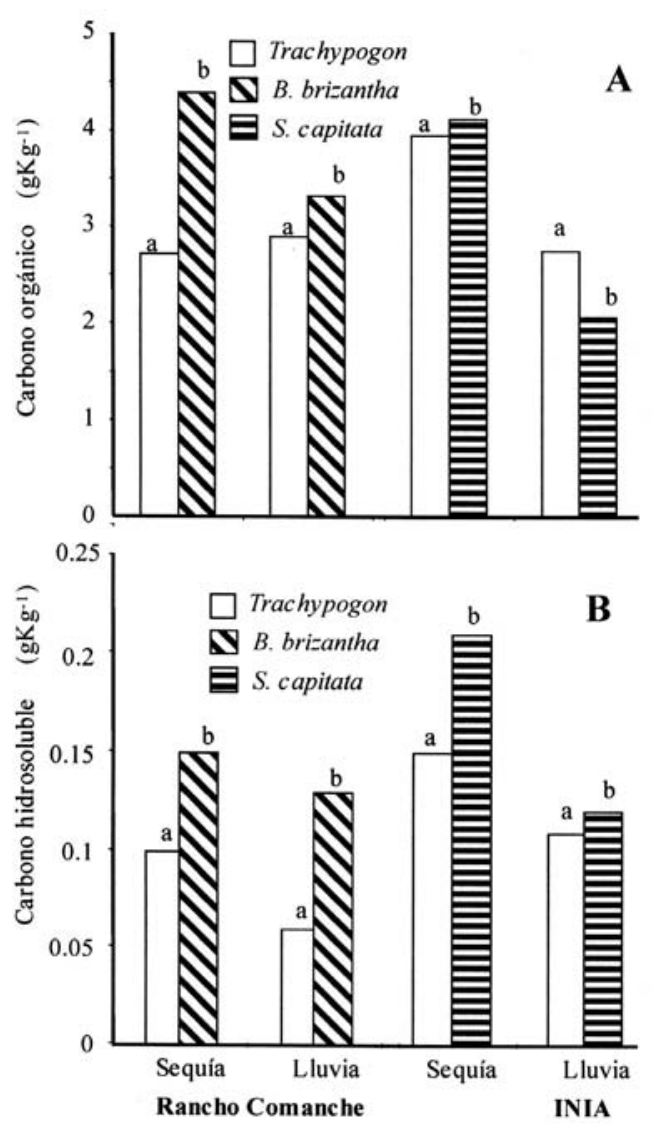

Fig. 1. Efecto del manejo agrícola sobre el Carbono orgánico (A) y el Carbono hidrosoluble (B) en las sabanas de los Llanos Orientales de Venezuela.

Fig. 1. Effect of soil managemt on the organic carbon (A) and water soluble carbon (B) in the Eastern Plain savannas of Venezuela.

lluvioso (Fig. 1 B). Un comportamiento similar, se observó en el INIA, en el cual el CHS, incrementó en un $30 \%$ bajo la cobertura de $S$. capitata en sequía y disminuyó en un $8.3 \%$ en el período húmedo.

\section{Parámetros bioquímicos}

Respiración basal. La respiración basal, desde el punto de vista ecológico es una medida de la actividad microbiana, la tasa de descomposición de la materia orgánica y de la calidad del carbono en el suelo (Anderson 1982, Brookes 1985, Saviozzi et al. 2001). Ésta resulta afectada por las condiciones ambientales, el sustrato disponible y los sistemas de manejo del suelo (Alvarez et al. 1995, Brookes 1995, Hayne 1999, Kandeler et al. 1999; Alvarez y Alvarez 2000, Palma et al. 2000), aunque existen controversias sobre el efecto de la fertilización inorgánica sobre este parámetro (Lovel et al.1995, Biederbeck et al. 1996 Svenson y Pell 2001).

Los valores de la respiración basal obtenidos en la sabana nativa variaron de 7.54 a $15.9 \mathrm{mg} \mathrm{C}-\mathrm{CO}_{2} \mathrm{~kg}^{-1} 24 \mathrm{hr}$ en $\mathrm{RC}$ y de a 8.0 a $13.4 \mathrm{mg} \mathrm{C}-\mathrm{CO}_{2} \mathrm{~kg}^{-1} 24 \mathrm{hr}$ en el INIA durante los períodos de sequía y lluvia respectivamente; mientras que en la sabana intervenida en $\mathrm{RC}$ la respiración basal varió de 7.5 a $16.8 \mathrm{mg} \mathrm{C}-\mathrm{CO}_{2} \mathrm{~kg}^{-1} 24 \mathrm{hr}$, y en el INIA fue de 11.9 a $23.7 \mathrm{mg} \mathrm{C}-\mathrm{CO}_{2} \mathrm{~kg}^{-1} 24 \mathrm{hr}$ durante estos períodos climáticos (Cuadro 1). Los valores obtenidos en esta investigación son más bajos que los reportados por otros autores para los llanos Orientales (Campos 1999) y Centrales de Venezuela (Hernández y López-Hernández 1996, 1998).

El Cuadro 3 muestra, que en RC la variación de los valores de la respiración están determinados exclusivamente por la variación temporal $(\mathrm{p}<0.001)$. En contraste, las variaciones de las tasas de respiración basal obtenidas en el INIA, están determinadas no sólo por este factor $(\mathrm{p}<0.001)$, sino también por el tipo de cobertura $(\mathrm{p}<0.001)$, y la interacción de ambos factores $(p<0.001)$ (Cuadro 3 y Fig. 2 A). En el INIA, durante los períodos de sequía y lluvia, la tasa respiratoria fue mayor bajo el cultivo de $S$. capitata.

Amonificación de la arginina. La AA, es un proceso intracelular el cual consiste en la liberación de amonio a partir de compuestos nitrogenados que son usados como fuentes de C y N (Alef y Kleiner 1986 a, b). Ésta ha sido empleada como una medida de la calidad del suelo (Sparling 1997) y de la actividad microbiana, ya que la acumulación y mineralización del $\mathrm{N}$ son procesos predominantemente 
biológicos (Alef et al. 1988, Franzluebbers et al. 1995, Lin y Brookes 1999, Bonde et al. 2001); Ésta también ha sido usada como un indicador del potencial existente en el suelo para llevar a cabo la mineralización del N (Alexander 1980).

En RC y el INIA la mineralización de la arginina estuvo significativamente determinada por la variabilidad temporal $(\mathrm{p}<0.001)$ (Cuadros 2 y 3 ). Estos valores variaron en la sabana nativa de RC de 1.1 a $3.7 \mu \mathrm{g} \mathrm{N}-\mathrm{NH}_{4}$ $\mathrm{g}^{-1} \mathrm{hr}^{-1}$ y para el cultivo de $B$. brizantha la variación fue de 1.6 a $2.1 \mu \mathrm{g} \mathrm{N}-\mathrm{NH}_{4} \mathrm{~g}^{-1} \mathrm{hr}^{-1}$ durante los períodos secos y húmedos, respectivamente (Cuadro 2). En el INIA se observó una tendencia similar de la AA; en la sabana nativa durante los períodos de sequía y lluvia, respectivamente, estos valores variaron de 1.0 - $1.7 \mu \mathrm{g} \mathrm{N}-\mathrm{NH}_{4} \mathrm{~g}^{-1} \mathrm{hr}^{-1}$, y en la sabana intervenida con $S$. capitata estos variaron de 1.4 a $1.9 \mu \mathrm{g} \mathrm{N}-\mathrm{NH}_{4} \mathrm{~g}^{-1} \mathrm{hr}^{-1}$, sin embargo, este parámetro en suelos de los Llanos Orientales también está determinado por el manejo agrícola $(\mathrm{p}<0.001)$ y por la interacción de estos factores $(p<0.001)$ (Cuadro 3 y Fig. $2 B)$. En $\mathrm{RC}$ la mayor actividad de la AA. se registró bajo la pastura de $B$. brizantha en sequía (1.61 $\left.\mu \mathrm{g} \mathrm{N}-\mathrm{NH}_{4} \mathrm{~g}^{-1} \mathrm{hr}^{-1}\right)$ y bajo la vegetación nativa en la temporada lluviosa (3.66 $\mu \mathrm{g} \mathrm{N}^{-N_{4}} \mathrm{~g}^{-1}$ $\mathrm{hr}^{-1}$ ), mientras que en el INIA la mayor tasa de mineralización del $\mathrm{N}$ ocurrió bajo la cobertura de $S$. capitata en ambos períodos climáticos.

\section{Hidrólisis del diacetato de fluorisceí-}

na. La hidrólisis del diacetato de fluorisceína (DAF) es usada en general como un indicador de la actividad hidrolítica, ya que ésta es hidrolizada por enzimas tales como proteasas, lipasas y esterasas. Es un parámetro que mide la actividad heterotrófica en el suelo (Schnürer y Roswall 1982, Taylor et al. 2002).

En RC la hidrólisis del DAF resultó afectada en forma significativa por la variabilidad temporal $(\mathrm{p}<0.001)$ (Cuadros 2 y 3), lo cual contrasta con los informes de Bandick y Dick (1999) y Dumontet et al. (2001), quienes afirman que la DAF es relativamente estable a la estación. Los valores de la DAF variaron en la sabana nativa de RC de 38.65 a $51.1 \mu \mathrm{g}$ de
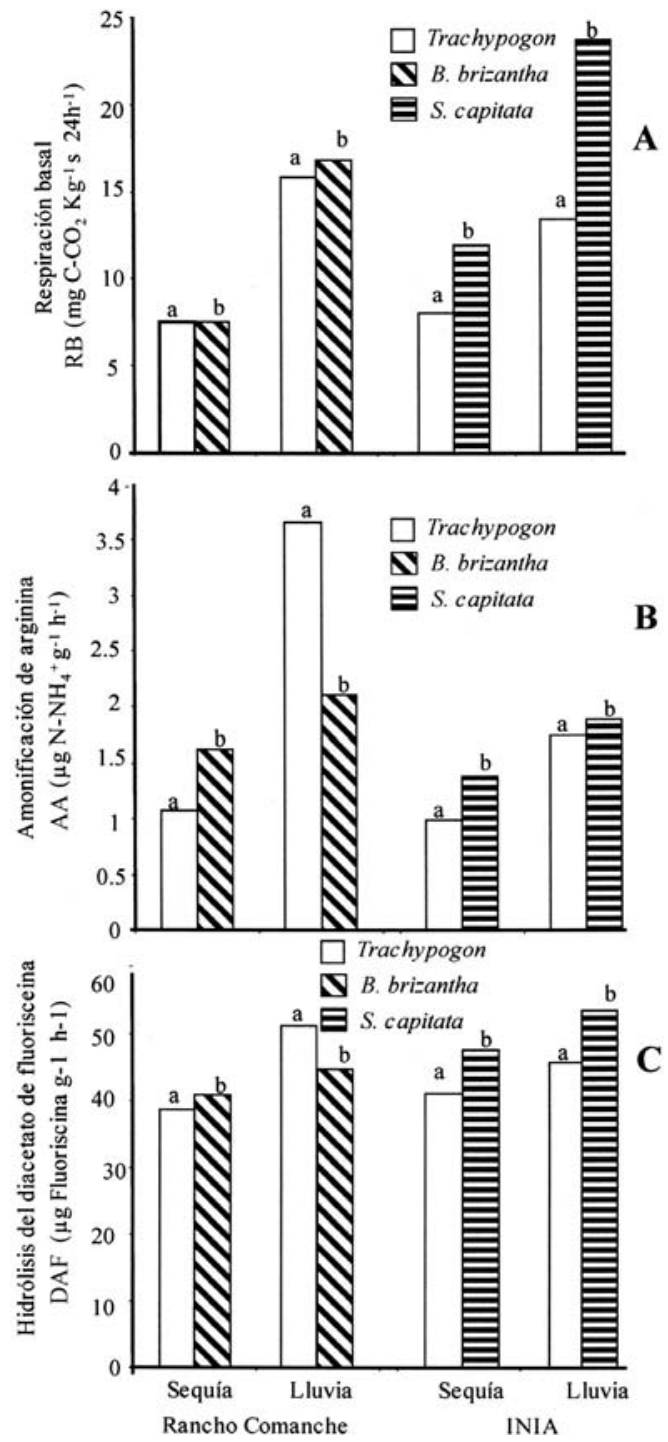

Fig. 2. Efecto del manejo agricola sobre: A) la respiración basal (RB), B) la amonificación de la arginina (AA), y C) la hidrólisis del diacetato de fluorisceina (DAF), en las sabanas de los Llanos Orientales de Venezuela.

Fig. 2. Effect of soil management on: A) basal respiration (RB), B) arginine ammonification (AA), and C) fluoriscein diacetate hydrolysis (DAF), in the Eastern Plain savannas of Venezuela.

fluorisceina $\mathrm{g}^{-1} \mathrm{hr}^{-1}$ y bajo la cobertura de $B$. brizantha, esta variación fue de 40.8 a $44.7 \mu \mathrm{g}$ de fluorisceina $\mathrm{g}^{-1} \mathrm{hr}^{-1}$ durante los períodos de lluvias y de sequía, respectivamente (Cuadro 
2). En el INIA la hidrólisis del DAF experimentó una variabilidad temporal similar a la de la AA (Cuadro 2). Esta incrementó en la sabana nativa de $41.1 \mathrm{a} 45.6 \mu \mathrm{g}$ de fluorisceina $\mathrm{g}^{-1} \mathrm{hr}^{-1}$ y bajo la pastura de $S$. capitata, la variación fue de 47.4 a $53.4 \mu \mathrm{g}$ de fluorisceina $\mathrm{g}^{-1} \mathrm{hr}^{-1}$ durante la temporada lluviosa y de sequía, respectivamente.

En RC el sistema de cobertura no ejerció un efecto significativo sobre la hidrólisis de la DAF; aunque la interacción variabilidad temporal x manejo del suelo, si ejerció un efecto significativo sobre la misma (Cuadro 3 y Fig. 2C). En contraste, en el INIA la cobertura afectó en forma significativa la hidrólisis de la DAF $(\mathrm{p}<0.001)$. En esta área la mayor actividad se observó bajo el cultivo de $S$. capitata, con un incremento con respecto a la sabana nativa del $13 \%$ en sequía y del $14.5 \%$ durante el período de lluvias.

\section{DISCUSIÓN}

Los valores del COT, aunque se encuentran por debajo del ámbito señalado para algunas sabanas de los Llanos Orientales (Campos 1999, Albornoz 1995 y Navas, 2000) y centrales de Venezuela (Paolini y Espana 1998, Hernández-Valencia y López-Hernández 1999, Hernández-Hernández y López-Hernández 2002), éstos están dentro del límite informado por Chapuis-Lardy et al. (2001) para el Cerrado del Brazil.

La disminución que experimentó el COT y el CHS en ambas zonas durante el período lluvioso, sugiere, que durante el mismo, una proporción significativa de éste, se pierde por lavado del suelo (Eaton 2001); lo cual es facilitado por la textura arenosa del mismo. Se ha informado que el incremento del CHS desde la superficie a capas profundas del suelo, representa una de las principales formas de translocación de nutrientes y compuestos energéticos a través de los diferentes compartimientos del ecosistema (Qualls y Haines 1992). Otra posible explicación, es que gran parte del carbono lábil presente en el suelo durante el período húmedo, sea mineralizado rápidamente por los microorganismos del suelo. Birch (1966) informó que regiones que presentan períodos de sequía, como es el caso de las sabanas, el rehumedecimiento del suelo con la temporada lluviosa induce a un incremento de la mineralización.

Respiración basal. A pesar del mayor contenido de CHS presente en el suelo durante la temporada seca (Cuadro 1), en términos generales, la respiración basal resultó aproximadamente 2 veces menor tanto en la sabana nativa como intervenida durante este período climático en ambas áreas de estudio, lo cual sugiere, que en este período gran parte de la población muere o permanece inactiva bajo las condiciones desfavorables del mismo, tal vez debido al estrés osmótico ocasionado por la baja disponibilidad del agua, o bien a la reducción del fenómeno de difusión (Harris 1981, Diaz-Raviña et al. 1988, Coyne 2000 y Marschner et al. 2002). Mientras que el incremento de este parámetro durante la temporada húmeda, está fuertemente determinado por el contenido de humedad del suelo. Ante mejores condiciones de humedad, el CHS es rápidamente mineralizado, y utilizado en la síntesis celular. Raich y Schlesinger (1992) informaron el efecto beneficioso de la precipitación sobre la mineralización del carbono. Así mismo, es probable que ante las condiciones más favorables existentes durante el período de lluvias, ocurra una mayor diversidad funcional de los microorganismos en contraste con el período de sequía, en la cual sobrevive sólo una parte de la población de la estación húmeda (Degens 1988, Marschner et al. 2002). El incremento de la diversidad de las comunidades microbianas del suelo incide sobre la actividad de uno o más procesos microbiológicos en el mismo (Kandeler et al. 1996). Este es un parámetro que muestra variaciones naturales dependiendo del sustrato disponible, la humedad y la temperatura (Alvarez et al. 1995 y Brookes 1995).

Las diferencias en magnitud del efecto de la cobertura sobre la respiración basal en $\mathrm{RC}$ y el INIA, especialmente durante la temporada lluviosa (Cuadro 2) podrían explicarse en parte 
por la historia de cultivo. Ésta indica, que la última vez en la cual el suelo de RC se sometió a labranza (arado y rastra) fue en 1995 antes de establecimiento del pasto $B$. brizantha, por lo que es posible, que en el transcurso de este período, las poblaciones microbianas hayan logrado un cierto estado de adaptación en el cual la tasa respiratoria llega a igualar a aquellas producidas por la flora microbiana de la sabana nativa. Se ha informado, que los ecosistemas llegan a alcanzar un estado de equilibrio cuando el manejo del suelo no se efectúa por largos períodos (Hassik 1994 b). Mientras que en el INIA, el efecto significativo causado por el cambio de cobertura sobre la respiración basal, especialmente durante el período de lluvias puede estar determinado por las labranzas recientes (arado y rastra) sufridas en 1998 para establecer un cultivo de C. rotundilfolium y luego en el 2000 previo a la introducción de $S$. capitata. Es probable, que estas recientes y frecuentes labranzas del suelo, asociadas al empleo de las rotativas anuales en los mismos, hayan provocado una mayor oxidación de la materia orgánica, lo cual se ve reflejado en una mayor tasa respiratoria. Así mismo, la mayor diversidad de manejos con rotación de cultivo realizados durante 1998 y el 2000 en esta zona, pueden alterar la estructura de las poblaciones microbianas, ya que se incorpora una mayor diversidad de sustratos de carbono, los cuales generalmente actúan como controladores primarios de las poblaciones microbianas del suelo (Díaz-Raviña et al. 1998, Tate 2000). La labranza y la incorporación de residuos incrementan la tasa respiratoria (Haynes 1999).

Otra posible explicación a las diferencias en las tasas respiratorias observadas bajo ambas coberturas, puede estar relacionada a la composición de los residuos de B. brizantha y $S$. capitata los cuales permanecen en la superficie del suelo una vez realizadas las rotativas anuales al inicio del período de lluvias; residuos que son metabolizados a diferentes tasas dependiendo de su contenido de C, N, S y lignina (Janzen y Kucey 1988). La fracción de los compuestos orgánicos presentes en los residuos de la leguminosa $S$. capitata, son de más fácil degradación que aquellos presentes en la gramínea $B$. brizantha (Ferreira y Costa 2003, Guenni et al. 2002). La calidad bioquímica de los sustratos orgánicos añadidos al suelo, constituye la clave que determina la tasa de su descomposición (Bending et al. 2002). Por otro lado, es importante señalar, que los exudados provenientes del sistema radicular de ambos tipos de cultivos podrían diferir en su composición, lo cual también puede alterar la estructura de la comunidad microbiana nativa (Lovell et al. 1995), y el perfil del nivel fisiológico de la comunidad presente en estos suelos (Garland 1996, Degens 1998).

Amonificación de la arginina. Rápidas respuestas de la AA al contenido de humedad del suelo han sido informadas por Bonde et al. (2001). La escasez de datos publicados para el trópico sobre este parámetro limita la comparación de los mismos. Sin embargo, los valores obtenidos en esta investigación se encuentran dentro de aquellos valores más bajos informados por Aleff y Kleiner (1987), Kaiser et al. (1992) Gumpala y Scow (1998), Lin y Brookes (1999) y Leiros et al. (2000) para diferentes suelos. La magnitud de estos valores sugiere que en los suelos de las sabanas de los Llanos Orientales de Venezuela, tanto en época de sequía como de lluvia predomina una flora microbiana que utiliza este aminoácido en menor grado (Dilly y Munch 1988).

El mayor potencial de mineralización del $\mathrm{N}$ en ambas áreas de estudio ocurrió en la temporada lluviosa, lo cual se corresponde con los incrementos experimentados por otros parámetros bioquímicos durante este período. Una tendencia estacional similar de la AA en estas sabanas, ha sido informada por Gumpala y Scow (1998).

Hidrólisis del diacetato de fluorisceína. Estos resultados confirman, que durante el período de lluvias ocurre una mayor actividad microbiana en estos suelos, lo cual está en concordancia con los incrementos experimentados por la AA y la respiración basal. 
A pesar de que no existen datos comparables de este parámetro para sistemas de pasturas tropicales, estos se encuentran dentro del límite informado por otros investigadores para suelos agrícolas bajo diferentes manejos (Burket y Dick 1998, Bandick y Dick 1999, Mendez et al. 1999, Taylor et al. 2002).

Nuestros resultados permiten sugerir que el clima, el manejo del suelo y el perfil del suelo de las sabanas de los Llanos Orientales de Venezuela son factores que afectan los parámetros bioquímicos estudiados, y que los períodos climáticos, provocan en ellos una gran variabilidad; lo cual confirma los señalamientos de Anderson (1994) quien sugirió que cualquier cambio en las condiciones medioambientales genera variaciones en el grado de actividad de los mismos.

\section{RESUMEN}

Se determinó el efecto de la variabilidad temporal y de las prácticas de manejo agrícola sobre las propiedades químicas y parámetros bioquímicos en un típico suelo Ultisol de pasturas nativas e introducidas de las sabanas orientales de los Llanos de Venezuela. La variabilidad temporal, el manejo agrícola y el efecto interactivo de ambos factores, afectaron las propiedades químicas y los parámetros bioquímicos. El carbono orgánico total e hidrosoluble experimentaron una significativa disminución durante la estación lluviosa. La respiración basal, la amonificación de la arginina (AA) y la hidrólisis del diacetato de fluorisceína (DAF) mostraron que en esas sabanas existe una baja actividad microbiana. La transformación de sabanas nativas a sistemas de pasturas de $B$. brizantha y $S$. capitata, incrementaron el contenido de materia orgánica en el suelo. La AA, y la respiración basal fueron indicadores sensibles, con los cuales se pueden estimar cambios en la calidad del suelo y reflejar el efecto causado por la interacción de los cambios temporales y las prácticas de manejo.

Palabras clave: amonificación, arginina, respiración basal, fluoresceína, prácticas de manejo, sabanas, variabilidad temporal, Venezuela.

\section{REFERENCIAS}

Albornoz, A., A. González \& O. Fuentes. 1995. Taller Aspectos Físicos de las sabanas Orientales y su Efecto sobre la Productividad. Fondo Nacional de Investigaciones Agropecuarias del Estado, Anzoátegui. Barcelona, España: 37 p.

Alef, K. \& D. Kleiner. 1987. Applicability of arginine ammonification as indicator of microbial activity in different soils. Biol. Fertil Soils. 5: 148-151.

Alef, K. \& D. Kleiner. 1986a. Arginine ammonification in soil samples. In The application of enzymatic and microbiological methods in soil analysis. Veröff. Landwertschchem. Bundesanstat Linz/Donau. 18: 163-168.

Alef, K. \& D. Kleiner. 1986b. Arginine ammonification a simple method to estimate microbial activity potentials in soils. Soil Biol. Biochem. 18: 233-235.

Alef, K. \& P. Nannipieri. 1995. Methods in applied soil microbiology and biochemitry. $340 \mathrm{p}$.

Alef, K., T.H. Beck, L. Zelles \& D. Kleiner. 1988. A comparison of methods to estimate microbial biomass and $\mathrm{N}$ - mineralization in agricultural and grassland soils. Soil Biol. Biochem. 20: 561-565.

Alexander, M. 1980. Introducción a la Microbiología del Suelo. AGT. México, DF. 483 p.

Alvarez, C.R. \& R. Alvarez. 2000. Short-term effects of tillage systems on active soil microbial biomass. Biol. Fertil. Soils. 31: 157-161.

Alvarez, R., R.A. Diaz, N. Barber, O.J. Santanatoglia \& L. Blotta. 1995. Soil organic carbon, microbial biomass and $\mathrm{C}-\mathrm{CO}_{2}$ production from three tillage systems. Soil Till Res. 33: 17-28.

Anderson, J.P.E.1982. Soil respiration. pp. 837-871. In A.L., Miller, R.H., Keeney, D.R. (eds). Methods of Soil Analysis, Part 2: Chemical and Microbiological Properties, Second ed. Society of Agronomy and Soil Science Society of America, Madison, Wisconsin, EEUU.

Anderson, T.H. 1994. Physiological analysis of microbial communities in soil: Applications and limitations. pp 67-76. In K. Ritz., J. Dighton \& K.E. Giller (eds.). Beyond the Biomass. Wiley, Nueva York, EEUU.

Anderson, T.H. \& K.H. Domsch. 1985. Determination of ecophysiological maintenance $\mathrm{C}$ requirements of soil microorganisms in a dormant state. Biol. Fertil. Soils. 1: $81-89$

Bandick, A.K. \& R.P. Dick. 1999. Field management effects on soil enzyme activities. Soil Biol Biochem. 31: 1471-1479.

Bending, G.D., M.K. Turner \& J.E. Jones. 2002. Interactions between crop residue and soil organic matter quality and the functional diversity of soil microbial; communities. Soil Biol. Biochem. 34: 1073-1082. 
Biederbeck, V.O., C.A. Campbell, H. Ukrainetz, D. Curtin \& O.T. Bouman. 1996. Soil microbial and biochemical properties after ten years of fertilization with urea and anhydrous ammonia. Can J. Soil Sci. 76: 7-14.

Birch, H.F. 1960. Nitrification in soils after different periods of dryness. Plant Soil. 12: 81-96.

Bonde, T.A., B.T. Christensen \& C. Cerri. 1992. Dynamics of soil organic matter as reflected by natural ${ }^{13} \mathrm{C}$ abundance in particle size fractions of forested and cultivated oxisols. Soil. Biol. Biochem. 24: 275-277.

Brookes, P.C. 1995. The use of microbial parameters in monitoring soil pollution by heavy metals. Biol. Fertil. Soils. 19: 269-279.

Brookes, P.C., A. Landman, A., G. Pruden \& D.S. Jenkinson. 1985. Chloroform fumigation and the release of soil nitrogen: a rapid direct extraction meted to measure microbial biomass nitrogen in soil. Soil Biol. Biochem. 17: 837- 842 .

Buckman, H.O. \& N. Brady. 1966. Naturaleza y propoiedades de los suelos. Unión Tipográfica .Hispano Americana. México, DF. 567 p.

Burket, J.Z. \& R.P. Dick. 1998. Microbial and soil parameters in relation to $\mathrm{N}$ mineralization in soils of diverse genesis under differing management systems. Biol. Fertil Soils. 27: 430-438.

Campos, G.A.J. 1999. Efecto de la siembra de Pinus caribea L. En las fracciones de materia orgánica de un suelo de sabana. Uverito-Edo. Monagas. Tesis de Licenciatura en Biología. Universidad Central de Venezuela, Caracas. 84 p.

Castillo, X. \& R.G. Joergensen. 2001. Impact of ecological and conventional arable management systems on chemical and biological soil quality indices in Nicaragua. Soil Biol. Biochem. 33: 1591- 1597.

Chapuis-Lardy, L., M. Brossad \& H. Quiquampoix. 2001. Assessing organic phosphorus status of Cerrado oxisols (Brazil) using ${ }^{31} \mathrm{P}-\mathrm{NMR}$ spectroscopy and phosphomonoesterase activity measurement. Can. J. Soil Sci. 81: 591-601.

Coyne, M. 2000. Microbiología del suelo: un enfoque exploratorio. Paraninfo, Madrid, España. 397 p.

Curl, E.A. \& B. Truelove. 1986. The Rhizosphere. Springer, Berlin, Alemania. 228 p.

Degens, B. 1998. A novel approach for assessing the pattern of catabolic potential of soil microbial communities, pp 206- 214. In H. Insam \& A. Rangger. (eds.). Microbial communities. Springer, Berlin, Alemania.
Delgado, E.F. 2001. Agricultura sostenible y mejoramiento de suelos de ladera. CIDIAT. Mérida, Venezuela. $200 \mathrm{p}$.

Diaz-Raviña, M., T. Carballas \& M.J. Acea. 1988. Microbial biomass and metabolic activity in four acid soils. Soil Biol. Biochem. 20: 817- 823 .

Dick, R.P. 1997. Soil enzyme activities as integrative indicators of soil health. p. 121-156. In C.E. Pankhurst, B.M. Doube \& V.V.S.R. Gupta (eds.). Biological indicators of soil health. CAB International, Walingford, Inglaterra.

Dick, W.A. \& M.A. Tabatabai. 1992. Potential uses of soil enzymes. pp. 95-127. In F.B. Metting Jr. (ed.). Soil Microbial Ecology: Applications in Agricultural and Environmental Management. Marcel Dekker, Nueva York, EEUU.

Dick, R.P. \& V.V.S.R. Gupta. 1994. A conceptual model for the role of abiontic soil enzymes in microbial ecology: a potential analogue for soil quality. pp 167-168. In C.E. Pankhurst, B.M. Double, V.V.S.R. Gupta \& P.R. Grace (eds.). Soil biota: management sustainable farming systems. CSIRO, Melbourne, Australia.

Dilly, O. \& J.C. Munch. 1988. Ratio between estimates of microbial biomass content and microbial activity in soils. Biol. Fertil. Soils. 27: 374-379.

Dorich, R.A. \& D.W. Nelson. 1983. Direct colorimetric measurements of ammonium in potassium chloride extracts of soils. Soil Sci. Soc. Am. J. 47: 833-836.

Doran, J.W.\& T.B. Parkin. 1994. Defining and assessing soil quality. pp 3-21. In J.W. Doran, D.C. Coleman, D.F. Bezdicek \& B.A. Stewart (eds.). Defining soil quality for sustainable environment. Soil Science Society of America special Publication $\mathrm{N}^{\circ}$ 35. SSSA, Madison. Wisconsin, EEUU.

Dumontet, S.A. Mazzatura, C. Cassici \& P. Perucci. 2001. Effectiveness of microbial indexes in discriminating interactive effects of tillage and crop rotations in a vertic ustorthens. Biol. Fertil. Soils. 34: 411-416.

Eaton, W.D. 2001. Microbial and nutrient activity in soils from three different subtropical forest habitats in Belize, Central America before and during the transition from dry to wet season. Appl. Soil Ecol. 16: 219-227.

Elliot, E.T. 1994. The potential use of biotic activity as indicators of productivity, sustainability and pollution. p. 250-256. In C.E. Pankhrust, B.M Doube, V.V.S.R. Gupta \& P.R. Grace (eds.). Soil Biota: management in sustainable farming systems. CSIRO, Melbourme, Australia. 
Fassbender, H.W. \& E. Bornemisza. 1987. Química de suelos, con énfasis en suelos de América Latina. Colección Libros y Materiales Educativos/IICA; N 81. San José, Costa Rica. 404 p.

Fauci, M.F. \& R.P. Dick. 1994. Microbial biomass as an indicator of soil quality: Effects of long-term management and recent soil amendments. In p. 229-234. Defining soil quality for a sustainable environment, SSSA Especial publication $\mathrm{N}^{\circ}$ 5. SSSA, Madison. Wisconsin, EEUU.

Franzluebbers, A.J., F.M. Hons \& D.A. Zuberer 1995. Tillage and crop effects on seasonal soil carbon and nitrogen dynamics. Soil Sci. Soc. Am. J. 59: 1618-1624.

Gillian A. \& H. Duncan. 2001. Development of a sensitive and rapid method for the measurement of total microbial activity using fluorescein diacetate (FDA) in a range of soils. Soil Biol. Biochem. 33: 943-951.

Gil-Sotres, F. 1997. El equilibrio bioquímico en los suelos. Implicaciones en el diagnóstico de la calidad del suelo. Trabajo de Investigación para concursar a una plaza de catedrático de la Universidad de Edafología y Química agrícola de la Universidad de Santiago de Compostela. 298 p.

Guenni, O., D. Marín \& Z. Baruch. 2002. Responses to drought of five Brachiaria species. I. Biomass production, leaft growth, root distribution, water use and forage quality. Plant Soil 243: 229-241.

Gunpala, N. \& K.M. Scow. 1998. Dynamics of soil microbial biomass and activity in conventional and organic farming system. Soil. Biol. Biochem. 30: 805-816.

Harris, R.F. 1981. Effect of water potential on microbial growth and activity. p. 23-95. In J.F. Parr, W.R. Gardner \& L.F. Elliot (eds.). Water Potential Pelations in Soil Microbiology. Soil Scie. Soc. Am. Special Publ. Number 9. Madison.

Hassink, J. 1994 a. Effects of soil texture and grassland management on soil organic $\mathrm{C}$ and $\mathrm{N}$ and rate of $\mathrm{C}$ and $\mathrm{N}$ mineralization. Soil Biol. Biochem. 26: 1221-1231.

Haynes, R.J. 1999. Size and activity of the soil microbial biomass under grass and arable management. Biol. Fertil. Soils. 30: 210-216.

Hernández, R.M. \& D. López-Hernández. 1996. Efecto de las prácticas agrícolas sobre algunas fracciones dinámicas de la materia orgánica. XIII Congreso latinoamericano del Suelo. Aguas de Lindoia, Brasil (CD ROM).

Hernández, R.M. \& D. López-Hernández. 1998. Mineralización del N y C microbiano y su inmovilización en la biomasa microbiana en agregados de un suelo tropical bajo dos tipos de labranza. Trabajo. XVI Congreso Mundial de la Ciencia del Suelo. Francia (CD ROM)

Hernández-Hernández, R.M. \& D. López-Hernández. 2002b. El tipo de labranza como agente modificador de la material orgánica: Un modelo para suelos de sabana de los llanos centrals. Interciencia 27: 529-535.

Hernández-Valencia, I. \& D. López-Hernández. 1999. Allocation of phosphorus in tropical savanna. Chemosphere 39: 199-207.

Holland, E.A. \& D.C. Coleman. 1987. Litter placement effect on microbial and organic matter dynamics in an agroecosystem. Ecology 68: 425-433.

Janzen, H.H \& R.M.N. Kucey. 1988. C, N and S mineralization of crop residues as influenced by crops species and nutrient regime. Plant Soil 106: 35-41.

Kaiser, E. A.,T. Muller, R. Joergensen, H. Insam \& O. Heinemeyer. 1992. Evaluation of methods to estimate the soil biomass and the relationship with soil texture and organic matter. Soil Biol. Biochem. 24: 675-683.

Kandeler, E., D. Tscherko \& H. Spiegel. 1999. Long-term monitoring of microbial biomass, $\mathrm{N}$ mineralization and enzyme activities of chernozem under different tillage management. Biol. Fertil. Soils. 28: 343-351.

Kandeler, E. \& K.E. Böhm. 1996. Temporal dynamics of microbial biomass, xylanse activity, $\mathrm{N}$-mineralization and potential nitrification in different tillage systems. Appl. Soil Ecol. 4: 181-191.

Kennedy, A.C. \& R.I. Papendick. 1995. Microbial characteristics of soil quality. J. Soil Sci. Water Conserv. 50: $243-248$.

Kieft, T.L. 1994. Grazing and plant-canopy effects on semiarid soil microbial biomass and respiration. Biol. Fertil. Soils. 18: 155- 162.

Leiros, M.C., C. Trasar-Cepeda, S. Seoane \& F. Gil-Sotres. 2000. Biochemical properties of acid soils under climax vegetation (Atlantic Oakwood) in an area of the European temperate-humid zone (Galicia, NW Spain): general parameters. Soil Biol. Biochem. 32: 733-745.

Lilienfein, J., W. Wilcke, S. do C Lima, L. Vilela, R. Thomas \& W. Zech. 2000. Nutrient concentration in soil solution of some Brazilian oxisols under conventional and no-tillage systems in the early part of the rainy season. Aust. J. Soil. Res. 38: 851-866.

Lin, Q. \& P.C. Brookes. 1999. Arginine ammonification as a method to estimate soil microbial biomass and microbial community structure. Soil Biol. Biochem. 31: 1985-1997. 
Lin, Q. \& P.C. Brookes. 1999. Comparison of substrate induced respiration, selective inhibition and biovolume measurement of microbial biomass and its community structure in unamended ryegrass-amended, fumigated and pesticide-treated soils. Soil Biol. Biochem. 31: 1999-2014.

Lovell, R.D., S.C. Jarvis \& R.D. Bardgett. 1995. Soil microbial biomass and activity in long-term grassland: Effects of management changes. Soil Biol. Biochem. 27: 969-975.

Marrero, L. 1964. Venezuela y sus Recursos, Cultural Venezolana, Caracas. 669 p.

MARNR. 1997. Atlas del Estado Monagas. Gobernación del Estado Monagas, Venezuela. 85 p.

Marschner, P., W. Marino \& R. Lieberei. 2002. Seasonal effects on microorganisms in the rhizosphere of two tropical plants in a polyculture agroforestry system in Central Amazonia, Brazil. Biol. Fertil Soils. 35: 68-71.

Mendez, I.C., A.K. Bandick, R.P. Dick \& P.J. Bottomley. 1999. Microbial biomass and activities in soil aggregates affected by winter cover crops. Soil Sci. Soc. Am. J. 63: 873-881.

Navas, M. 2000. Alternativas para el establecimiento, manejo y recuperación de pasturas en sabanas bien drenadas del estado Anzoátegui. Tesis de Magíster Scientiarum en Ciencias del Suelo. Universidad Central de Venezuela, Caracas, Venezuela. 77 p.

Palma, R.M., N.M. Arrigo, M.J. Saubidet \& M.E. Conti. 2000. Chemical and biochemical properties as potential indicators of disturbances. Biol. Fertil. Soils. 32: 381-384.

Paolini, J. \& Z.M. España. 1998. Acid phosphomonoesterase activity in soils under savanna vegetation of Venezuela. $16^{\text {th }}$ World Congress of Soil Science. Montepelier, Francia (CD ROM).

Qualls, R.G. \& B.L. Haines. 1992. Biodegradability of dissolved organic matter in forest through fall, soil solution, and stream water. Soil Sci. Soc. Am. J. 56: 578-586.

Raich, J.W. \& W.H. Schlesinger. 1992. The global carbon dioxide flux in soil respiration and its relations to vegetation and climate. Tellus 44: 81-99.

Šantrùèková, H. \& M. Straškraba. 1991. On the relationship between Specific respiration activity and microbial biomass in soils. Soil. Boil. Biochem 23: 525-532.

Saviozzi, A., R. Levi-Minzi, R. Cardelli \& R. Riffaldi. 2001. A comparison of soil quality in adjacent cultivated, forest and native grassland soils. 233: 251-259.

Schnürer, J. \& T. Rosswall. 1982. Fluorescein diacetate hydrolysis as a measurement of total microbial activity in soil and litter. Appl. Envirom. Microbiol. 43: 1256-1261.

Sparling, G.P. 1997. Soil microbial biomass, activity and nutrient cycling as indicators of soil health. p. 67-119. In C.E. Pankhurst; B.M. Doube \& V.V.S.R. Gupta (eds.). Biological Indicators of Soil Heath. CAB. International.

Sparling,G.P. \& A.W. West. 1989. Importance of soil water content when esmating soil microbial $\mathrm{C}, \mathrm{N}$ and $\mathrm{P}$ by fumigation-extraction methods. Soil Biol. Biochem. 21: $245-253$.

Srivastava, S.C. \& J.S Singh 1988. Carbon and phosphorus in the soil biomass of some tropical soils of India. Soil Biol. Biochem. 20: 743-747.

Svensson, K. \& M. Pell. 2001. Soil microbial test for discriminating between different cropping systems and fertilizer regimes. Biol. Fertil. Soils. 33: 91-99.

Tate III, R.L. 2000. Soil Microbiology. Wiley, Nueva York York, EEUU. p. 508.

Taylor, J.P., B. Wilson, M.S. Mills \& R.G. Burns. 2002. Comparison of microbial numbers and enzymatic activities in surface soils and subsoils using various techniques. Soil Biol. Biochem. 34: 387-401.

Vinacua, B.V. 1997. Análisis Estadísticos con SPSS para Windows. McGraw Hill, Madrid, España. 297 p.

Walkley, A. \& I.A. Black. 1934. An examination of Degtjareff method for determining soil organic matter, and a proposed modification of the chromic acid titration method. Soil. Sci. 37: 29-38.

\section{REFERENCIAS DE INTERNET}

Ferreira, M.B. \& N.M. Sousa Costa. 2003. Stylosantes macrocephala. (www.fao.org./ag/AGP/ AGPC/doc/Gbase/ DATA/Pf000495.HTM, consultado enero 2006). 
